\title{
The role of far-field intravascular ultrasound in transcatheter aortic valve replacement
}

\author{
Ahmed Hassanin 1* (0), Hasan Ahmad', Massoud Leesar² and Diaa Hakim 3,4
}

\begin{abstract}
Precise and accurate characterization of the aortic valve complex is a vital step in the procedure planning for transcatheter aortic valve replacement (TAVR). Far-field intravascular ultrasound (IVUS) is a novel technology that can be utilized to assess aortic valve annulus and predict paravalvular leak, with comparable results to multi-detector computed tomography - the current gold standard in the preprocedural planning in TAVR. Far-field IVUS carries the advantage of minimal contrast use and lower radiation exposure. In this commentary, we describe two cases of farfield IVUS use during TAVR procedures and review its role as a complementary tool to current the imaging modalities used in TAVR.
\end{abstract}

Keywords: Far-field intravascular ultrasound, Transcatheter valve replacement, TAVR, TAVI, Aortic stenosis, Aortic valve

\section{Background}

Transcatheter aortic valve replacement (TAVR) has revolutionized the treatment of patients with severe aortic stenosis. It has shown superior short and intermediateterm outcomes compared to the surgical aortic valve (SAVR) $[1,2]$. Imaging modalities in TAVR are designed to accurately assess the aortic valve complex to select the appropriate size of the valve implant and create a vascular roadmap to identify potential obstacles to procedural success. Multi-detector computed tomography (MDCT) plays a central role in procedure planning by providing high spatial resolution of the valve morphology, dimensions, and pathological characteristics such as calcification, deployment angle, and anatomy of the peripheral arteries [3]. The role of transesophageal echocardiography (TEE) in TAVR has decreased over time. It is mainly limited to intraoperative imaging to assess valve positioning, expansion and detection of paravalvular aortic regurgitation (PVR) [4].

Intravascular ultrasound (IVUS) is a widely used imaging tool to guide coronary percutaneous interventions

\footnotetext{
*Correspondence: dr.hassanein@gmail.com

${ }^{1}$ Department of Cardiology, Westchester Medical Center and New York Medical College, Valhalla, NY, USA

Full list of author information is available at the end of the article
}

[5, 6]. IVUS imaging of complex coronary lesions and for stent optimization is recommended in chronic total occlusions and left main coronary artery interventions. Additionally, IVUS is utilized in peripheral interventions and is considered the gold standard imaging modality for measuring the luminal diameter of the aorta and selecting appropriate landing zones for endografts [7]. Recently published studies have reported the adaptation of a far field IVUS imaging technology to aortic valve disease. This includes sizing of the the aortic annuals, implant size selection and in predicting paravalvular regurgitation (PVR). This commentary report describes two cases of far-field IVUS use during TAVR procedures and reviews its role as a complementary imaging modality to MDCT and TEE.

\section{Cases \\ Case 1}

An 89-year-old female with advanced emphysema and known severe calcific aortic stenosis presented for evaluation of chest pain and syncope. After excluding other etiologies for her presentation, aortic valve replacement was deemed appropriate. The patient's society of thoracic surgeons (STS) risk score was 8.4\%, making her a highrisk candidate for SAVR, so the patient was referred for 
TAVR evaluation. MDCT image quality was suboptimal due patient's inability to hold her breath and movement artifact. Cardiac CT measurements revealed annular diameters of $19 \times 24 \mathrm{~mm}$ and annulus surface area of $342 \mathrm{~mm}^{2}$ with a mean annular diameter of $21.5 \mathrm{~mm}$. TEE was considered for further aortic annular assessment, but the patient's advanced emphysema was a relative contraindication for sedation given the high risk for requiring endotracheal intubation. The decision was made to have the patient undergo intraprocedural aortic valve imaging by far-field IVUS using a $15 \mathrm{MH}$ Atlantis catheter interfaced with i-Lab imaging systems (Boston Scientific, Natick, Massachusetts). After obtaining femoral arterial access, a pigtail catheter was advanced over the wire into the ascending aorta and placed at the non-coronary cusp soft tip. Subsequently, a straight wire was used to cross over the aortic valve to the left ventricle, and the catheter was then exchanged for an extra stiff wire. The IVUS catheter was advanced over the extra-stiff guidewire to the left ventricle. An automatic pullback IVUS run at a speed of $0.5 \mathrm{~mm} / \mathrm{s}$ from the left ventricular outflow tract (LVOT) to the aortic root was undertaken. To enhance the visibility of the annulus and adventitial borders, the depth of IVUS penetration was increased to $50 \mathrm{~mm}$ and $2 \mathrm{~mL}$ agitated contrast media was injected through the lumen of the IVUS catheter. The cross-sectional area of the aortic valve annulus was obtained by planimetry of the circumference at the level of attachment of the aortic leaflet with the LVOT at its nadir point (most proximal to the LVOT). Figure 1 demonstrates reconstructed cross-sectional and longitudinal IVUS images obtained from the pullback of the IVUS catheter

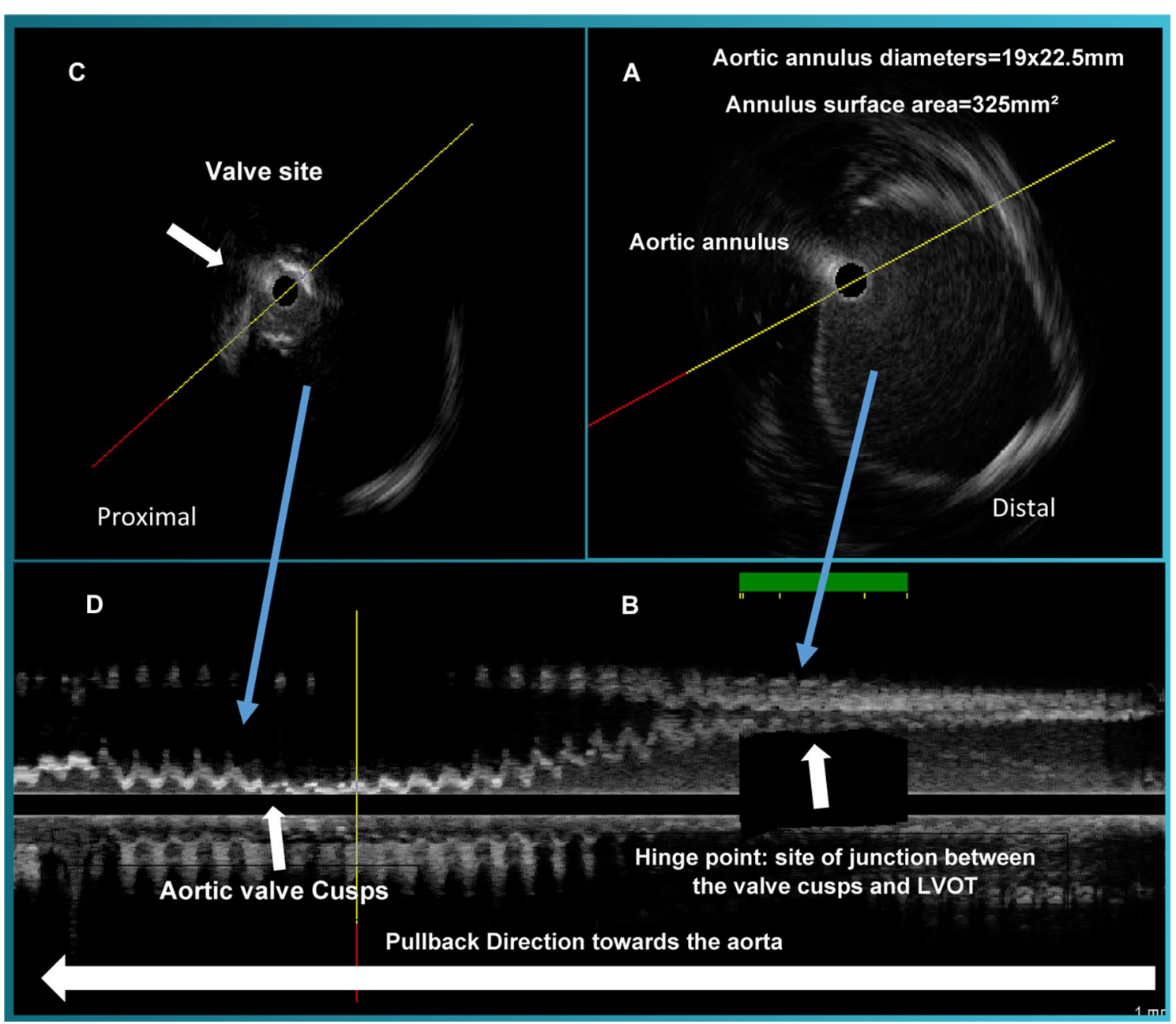

Fig. 1 Intravascular ultrasound measurements of the aortic annulus diameters and surface area. The pullback is shown from right to left. A The IVUS image of the aortic annulus, the annulus area was $325 \mathrm{~mm}^{2}$; B corresponding longitudinal IVUS image at the site of junction between LVOT and the aortic cusps; C calcified aortic valve with signal drop-out; D corresponding longitudinal IVUS image of aortic valve cusps 
from the LVOT to the aortic root. This area is the hinge point. IVUS measurements for the minimum, maximum, average annular diameters and annular surface area were $19 \mathrm{~mm}, 22 \mathrm{~mm}, 20.5 \mathrm{~mm}$, and $325 \mathrm{~mm}^{2}$, respectively, and correlated well with MDCT. A $23 \mathrm{~mm}$ Edward Sapien XT valve was successfully implanted with only trivial PVR. The post-procedure hospital course was uneventful, and the patient was discharged on post procedure day 3 .

\section{Case 2}

87-year-old female patient with hypertension and chronic kidney disease (CKD) stage IV presented with worsening New York Heart Association (NYHA) class III dyspnea symptoms. The patient was determined to have severe calcific aortic stenosis with an aortic valve area of $0.7 \mathrm{~cm}^{2}$, and a mean gradient of $42 \mathrm{mmHg}$ on transthoracic echocardiogram. Her STS risk score was estimated to be $8.1 \%$. After excluding other etiologies for the patient's symptoms, TAVR was deemed appropriate. MDCT was done without contrast given the patient's advanced CKD and the measurements revealed annular diameters of $19.5 \times 23.5 \mathrm{~mm}$, mean annular diameter of $22 \mathrm{~mm}$, and annulus surface area of $361 \mathrm{~mm}^{2}$. During the patient's pre TAVR left cardiac catheterization, angiography suggested moderate stenosis and calcification of both femoral arteries, so far-field IVUS was used to better assess the femoral arteries and demonstrated only mild stenosis. To further evaluate the aortic valve annulus, the IVUS catheter was then advanced over the wire to the aortic root. The IVUS measurements for the minimum, maximum, average annular diameters and annular surface area were $18.7 \mathrm{~mm}, 23.4 \mathrm{~mm}, 21.1 \mathrm{~mm}$, and 346 $\mathrm{mm}^{2}$, respectively, similar to the measurement obtained on MDCT. TEE measurements obtained during the TAVR procedure showed an average annulus diameter of 19.5 and annulus surface area $358 \mathrm{~mm}^{2}$, which correlated well with measurements obtained by MDCT and IVUS. Figure 2 demonstrates comparative measurements of the aortic root obtained by the three imaging modalities. Given the anatomical evaluations provided by MDCT, TEE and IVUS, a $23 \mathrm{~mm}$ Edward Sapien XT was selected. The valve was successfully implanted with only trivial PVR. The patient's hospital stay was without complications, and she was discharged on postoperative day 2 .

\section{Discussion}

MDCT is the gold stand for pre TAVR assessment of the aortic valve complex and assessing the iliofemoral vasculature for access consideration [3]. As compared to Two dimensional (2D) TEE, MDCT provides a more accurate assessment of the aortic annulus measurements and does not require sedation $[4,8]$. The reliance on intraprocedural 2D TEE in TAVR has steadily declined over the past 5 years as conscious sedation became the standard approach. It has been associated with shorter hospital stay and a trend for lower mortality compared to general anesthesia [9]. Nevertheless, the quality of MDCT may occasionally be limited due to partial volume-averaging effects (blooming), heart/ lung motion, arrhythmias, and patient motion [3], as was the case with the first patient presented. On the other hand, among patients with advanced CKD, the use of IV contrast for assessment of the iliofemoral arteries may be relatively contraindicated due to the increased risk of renal failure.

Far-field IVUS is a novel tool in the armamentarium of structural interventional cardiologists. Far-field IVUS has a maximum imaging diameter of $50 \mathrm{~mm}$ compared to a $20 \mathrm{~mm}$ diameter in traditional coronary IVUS catheters, which allows for adequate evaluation of the aortic valve complex. The use of IVUS in TAVR was first reported by Roy et al. in 2013 [10]. A case series in 2016 demonstrated an excellent correlation between aortic valve measurements obtained by MDCT and IVUS [11]. More recently, in 2017, Hakim et al. investigated the role of large-field IVUS vs. MDCT and 2D TEE for annular sizing and predicting PVR in 50 consecutive patients undergoing TAVR [12]. In their study, far-field IVUS demonstrated similar aortic annular diameters and area measurements compared to MDCT and performed as well in predicting PVR. There were strong correlations between IVUS and MDCT annular areas $(r=0.87, P<0001)$ and mean diameters $(r=0.73, P<0.0001)$. 2D TEE underestimated aortic annular diameter and did not correlate well with MDCT or IVUS. More recently, far-field IVUS use in TAVR has also been reported for the intraprocedural assessment of implanted valve frame geometry and leaflets mobility when under expansion is suspected [13].

Far-field IVUS has a strong correlation with MDCT and invasive angiography in the assessment of the iliofemoral arteries [14]. It carries the advantage of minimal contrast use and lower radiation exposure when performed at the- time of the routine pre-TAVR hemodynamic and coronary assessment as demonstrated in case number 2. Moreover, the data can be interpreted in real time, and are also a valuable adjunct to CTA in patients with borderline femoral access diameters or considerable CTA artifacts.

Far-field IVUS has a few limitations that should be acknowledged. If the IVUS catheter is not centralized, the images can be biased to one side, leading to oblique sections, and oversizing the aortic annulus. To avoid eccentric catheter position and motion artifacts, the IVUS catheter should be advanced over a stiff guidewire and measure the eccentricity index. If motion artifact is present the IVUS run can be repeated. 

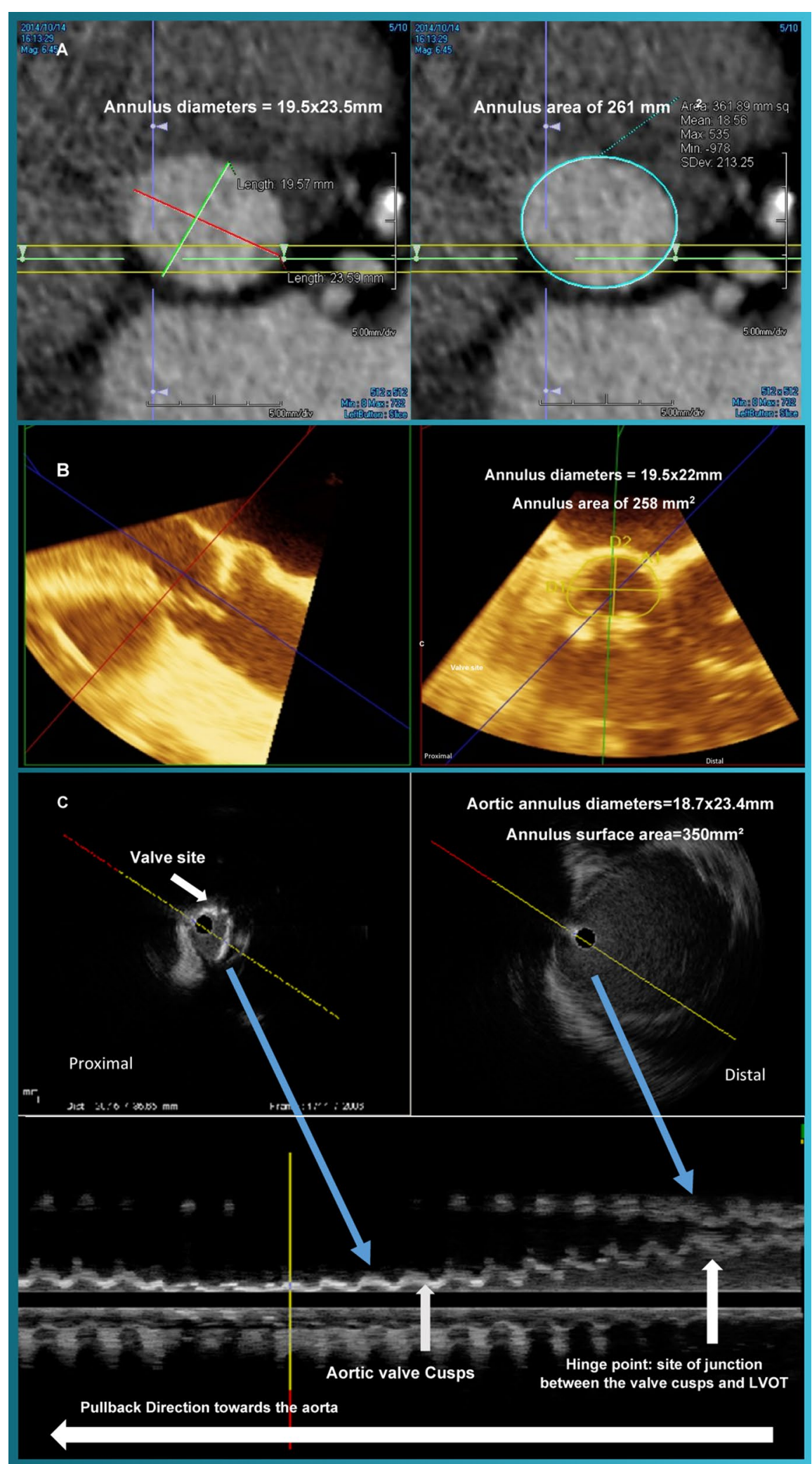

Fig. 2 Comparative measurements of the aortic valve annulus obtained by MDCT, 3D TEE and far field IVUS. Panel A MDCT measurements of the aortic valve annulus; Panel B 2D TEE measurements of the aortic valve annulus; Panel C far field IVUS measurements of the aortic valve annulus 
The IVUS catheter that was used during the cases was not primarily manufactured to visualize the aortic valve complex, so an IVUS catheter with better resolution can help in the assessment of the valve cusps, annulus, and PVR. The additional cost of utilizing the far-field IVUS catheter should also be considered.

\section{Conclusion}

Far-field IVUS use in TAVR is a novel technology that could be used to assess aortic annulus and predict PVR. The technology can help assess leaflets' mobility when a valve under expansion is suspected. Far-field IVUS can also minimize contrast use in patients with advanced CKD who are undergoing a pre TAVR evaluation.

\section{Abbreviations}

TAVR: Transcatheter aortic valve replacement; SAVR: Surgical aortic valve replacement; MDCT: Multi-detector computed tomography; TEE: Transesophageal echocardiography; PVR: Paravalvular aortic regurgitation; IVUS: Intravascular ultrasound; STS: Society of Thoracic Surgeons; LVOT: Left ventricular outflow tract; CKD: Chronic kidney disease; NYHA: New York Heart Association; 2D: Two dimensional.

\section{Acknowledgements}

Not applicable.

\section{Authors' contributions}

All authors have read and approved the final manuscript. A.H. and D.H formulated the commentary's idea and wrote the first draft. H.A. and M.L. contributed to the scientific content of the manuscript and edited the final version of the manuscript.

\section{Funding}

Not applicable.

\section{Availability of data and materials}

Not applicable.

\section{Declarations}

Ethics approval and consent to participate

The cases presented are de-identified and adequately anonymized and thus an ethics approval is not required or necessary.

\section{Consent for publication}

Written informed consent was granted by patients.

\section{Competing interests}

The authors do not have competing interests to declare.

\section{Author details}

1 Department of Cardiology, Westchester Medical Center and New York Medical College, Valhalla, NY, USA. ${ }^{2}$ Department of Cardiology, University of Alabama at Birmingham, Birmingham, AL, USA. Intravascular and Cardiac Imaging Core Laboratory, Brigham and Women's/Harvard School of Medicine, Boston, MA, USA. ${ }^{4}$ Department of Cardiology, Suez Canal University, Ismailia, Egypt.

Received: 8 June 2021 Accepted: 2 November 2021

Published online: 16 November 2021

\section{References}

1. Mack MJ, Leon MB, Thourani VH et al (2019) Transcatheter aortic-valve replacement with a balloon-expandable valve in low-risk patients. N Engl J Med 380(18):1695-1705. https://doi.org/10.1056/NEJMoa1814052

2. Popma JJ, Deeb GM, Yakubov SJ et al (2019) Transcatheter aortic-valve replacement with a self-expanding valve in low-risk patients. N Engl J Med 380(18):1706-1715. https://doi.org/10.1056/NEJMoa1816885

3. Blanke P, Weir-McCall JR, Achenbach S et al (2019) Computed tomography imaging in the context of transcatheter aortic valve implantation (TAVI)/transcatheter aortic valve replacement (TAVR): an expert consensus document of the society of cardiovascular computed tomography. JACC Cardiovasc Imaging 12(1):1-24. https://doi.org/10.1016/j.jcmg.2018. 12.003

4. Bleakley C, Monaghan MJ (2018) The pivotal role of imaging in TAVR procedures. Curr Cardiol Rep 20(2):9. https://doi.org/10.1007/ s11886-018-0949-z

5. Shammas NW, Radaideh Q, Shammas WJ, Daher GE, Rachwan RJ, Radaideh Y (2019) The role of precise imaging with intravascular ultrasound in coronary and peripheral interventions. Vasc Health Risk Manag 15:283-290. https://doi.org/10.2147/NHRM.S210928

6. Cereda A, Garascia A, Sormani P et al (2016) Embolic myocardial infarction due to coronary artery aneurysm in a patient with Loeys-Dietz syndrome. Eurolntervention 12(1):61. https://doi.org/10.4244/EIJV12I1A11

7. Wallace GA, Starnes BW, Hatsukami TS, Sobel M, Singh N, Tran NT (2015) Intravascular ultrasound is a critical tool for accurate endograft sizing in the management of blunt thoracic aortic injury. J Vasc Surg 61(3):630635. https://doi.org/10.1016/j.jvs.2014.10.014

8. Bleakley C, Monaghan M (2020) 3D transesophageal echocardiography in TAVR. Echocardiography 37(10):1654-1664. https://doi.org/10.1111/echo. 14777

9. Butala NM, Chung M, Secemsky EA et al (2020) Conscious sedation versus general anesthesia for transcatheter aortic valve replacement: variation in practice and outcomes. JACC Cardiovasc Interv 13(11):1277-1287. https://doi.org/10.1016/j.jcin.2020.03.008

10. Roy DA, Bhamra-Ariza P, Asherson A, Sharma R, Jahangiri M, Brecker S (2013) First-in-man use of aortic valve ultrasound for assessment of aortic valve anatomy pre- and post-transcatheter aortic valve implantation. JACC Cardiovasc Interv 6(6):634-635. https://doi.org/10.1016/j.jcin.2012. 11.019

11. de Cillis E, Dachille A, Giardinelli F, Acquaviva T, Bortone AS (2016) Accuracy of intravascular ultrasound evaluation for the assessment of native valve measures in patients undergoing TAVl: preliminary results. Surg Technol Int 29:201-206

12. Hakim D, Ghimire G, Alli OO et al (2017) Large-field intravascular ultrasound for annular sizing and predicting paravalvular regurgitation during TAVR: comparisons with multidetector computed tomography and transoesophageal echocardiography. Eur Heart J Cardiovasc Imaging 18(12):1404-1413. https://doi.org/10.1093/ehjci/jew322

13. Kalinczuk L, Stoklosa P, Dabrowski M et al (2021) Nonuniform expansion of the LOTUS Edge intra-annular transcatheter aortic valve seen on intravascular ultrasound as a mechanism of prosthesis-patient mismatch. Kardiol Pol 79(2):203-204. https://doi.org/10.33963/KP.15752

14. Essa E, Makki N, Bittenbender P et al (2016) Vascular assessment for transcatheter aortic valve replacement: intravascular ultrasound compared with computed tomography. J Invasive Cardiol 28(12):E172-E178

\section{Publisher's Note}

Springer Nature remains neutral with regard to jurisdictional claims in published maps and institutional affiliations. 